\title{
Spin transitions in time-dependent regular and random magnetic fields
}

\author{
V. L. Pokrovsky ${ }^{1,2}$ and N. A. Sinitsyn ${ }^{1}$ \\ ${ }^{1}$ Department of Physics, Texas A\&M University, College Station, Texas 77843-4242, USA \\ ${ }^{2}$ Landau Institute for Theoretical Physics, Chernogolovka, Moscow Distr, 142432, Russia
}

(Received 30 September 2003; published 19 March 2004)

\begin{abstract}
We study the transition between Zeeman levels of an arbitrary spin placed into a regular time-dependent magnetic field and a random field with the Gaussian distribution. One component of the regular field changes its sign at some moment of time, whereas another component does not change substantially. The noise is assumed to be fast. In this assumption we find analytically the ensemble average of the spin density matrix and its fluctuations.
\end{abstract}

DOI: 10.1103/PhysRevB.69.104414

PACS number(s): 02.50.Ey

\section{INTRODUCTION}

Nanomagnets $\mathrm{Fe}_{8}$ and $\mathrm{Mn}_{12}$ attracted much attention in the last decade. It was shown experimentally that they realized a single molecule quantum hysteresis. ${ }^{1}$ Some features of the hysteresis have been predicted in many theoretical works. ${ }^{2-4}$ In particular, it is commonly accepted that plateaus on the hysteresis loop are due to the Landau-Zener (LZ) transitions ${ }^{5,6}$ at avoided crossing of Zeeman-split spin levels in the crystal field of molecular environment. This point of view is strongly supported by the experimental observation ${ }^{7}$ of theoretically predicted ${ }^{8}$ oscillations of the transition matrix elements vs. magnetic field applied in the hard direction. However, it was indicated earlier ${ }^{4}$ that the hyperfine interaction with the numerous nuclei in the molecule is comparatively large and can remarkably complicate a simple LZ picture of transition. The reason why it did not happen at a specific experimental setup $^{7}$ was recently explained theoretically. ${ }^{9}$ It is indicated in the same work that at a smaller rate of the field sweeping the violation of the simple LZ formula seem to be unavoidable. The nuclear spins provide not only a random static field which changes locally Zeeman splitting and create a nonorthogonality. Their fluctuations at a little higher temperature become fast and can be considered as a random time-dependent magnetic field acting on the spin. Another source of the noise is the interaction with phonons ${ }^{10}$ which produces random, time-dependent anisotropy.

Recently several nanomagnets with cubic or almost cubic symmetry were synthesized. One of them $\mathrm{Mo}_{6} \mathrm{Mn}_{9}$ (Ref. 11) has spin $51 / 2$ (this value can be comparatively easy varied by simple substitutions). Another nanomagnet $\left[\text { (trifos) } \operatorname{Re}(\mathrm{CN})_{3}\right]_{4}[\mathrm{CoCl}]_{4},{ }^{12}$ which we will abbreviate as $\mathrm{Re}_{4} \mathrm{Co}_{4}$, has a smaller spin $S=6$. So far no effects of anisotropy were found in magnetic measurements even at temperature about $1 \mathrm{~K}$. It shows that the cubic anisotropy is weak, as expected. Thus, the cubic nanomagnets may be the best realization of a large free spin. Being placed into a varying magnetic field $B_{z}(t)$ along the $z$ axis and a small constant field $B_{x}$ in the $x$ direction, such a spin performs quantum transitions between the states of the Zeeman multiplet. The essential difference between this situation and the standard Landau-Zener (LZ) problem ${ }^{5,6}$ is that at $B_{z}(t)=0$ all $2 S$ +1 states of the Zeeman multiplet cross simultaneously, whereas Landau and Zener considered only the two-level crossing equivalent to $S=1 / 2$. An extension of the LZ theory to the case of higher spins was proposed by Hioe. ${ }^{13} \mathrm{He}$ proved that the transition probabilities depend only on $S$ and the dimensionless LZ parameter

$$
\gamma=\sqrt{g \mu_{B} B_{x}^{2} / 4 \hbar \dot{B}_{z}},
$$

where $g$ is the Lande factor, $\mu_{B}$ is the Bohr's magneton, and $\dot{B}_{z}$ is the time derivative (sweeping rate) of the magnetic field taken at the diabatic level crossing, i.e., at the moment when $B_{z}(t)$ turns into zero. The transition probabilities display very interesting oscillations versus the parameter $\gamma$ and difference of projections $m$ and $m^{\prime}$ of the orbital moment in the initial and final states. These oscillations, which were not analyzed in the work, ${ }^{13}$ originate from the quantum interference of different Feynman paths leading from $m$ to $m^{\prime}$, which does not exist in the genuine LZ problem. ${ }^{14}$ Thus, the cubic nanomagnets are simple, but nontrivial physical objects. The quantum tunneling in these objects is worthwhile to study. As shown for other nanomagnets, the interaction with nuclear spins is essential for the dynamics. Though the nuclear relaxation times are sufficiently long in microscopic scale (typically milliseconds), the characteristic time of the LZ process $\tau_{\mathrm{LZ}} \sim B_{x} / \dot{B}_{z}$ may be even longer. Then the field of nuclear spins can be considered as a fast Gaussian noise. In the opposite case this field should be considered as a quasistatic random field. Such a case was studied theoretically by Prokof'ev and Stamp. ${ }^{4}$

The purpose of our article is to study the influence of the fast noise onto probabilities of transitions between the states of Zeeman multiplet for a free spin $S$ in the presence of a regular time-dependent field, the same as that in the LZ or Hioe problem. A special case of this problem for $S=1 / 2$, when the regular field $B_{x}$ is zero and transitions are completely determined by nondiagonal elements of the random field, solved earlier by Kayanuma, ${ }^{15}$ who found average values of the transition probabilities. In our previous work ${ }^{16}$ we have solved the same problem for regular and random fields acting together. In this work we extend our results for higher spins, find the average nondiagonal matrix elements of the density matrix, and calculate the fluctuations of all these values, which are determined to be strong. These calculations became possible due to high symmetry of the problem. A 
proper group-theoretical treatment allows us to deal with standard objects, which we call Bloch tensors. They are a generalization of the well-known Bloch vector for spin $1 / 2$ problem.

The plan of the article is as follows: In Sec. II we remind the Hioe solution and analyze in some details the oscillations of the transition probabilities. In Sec. III we formulate equations for the density matrix and reduce them to equations for the Bloch tensors, which we define in the same section. In Sec. IV we consider the fast noise acting on a two-level system. Though this problem was considered in our previous article,${ }^{16}$ it is important to give a simple analysis of the time scales and to calculate the average transition matrix and fluctuations, which will serve as a basis for the spin- $S$ problem. Section V is dedicated to the solution of the same problem for general spin. In Sec. VI, returning to two-level system (spin 1/2), we discuss the limit of a strong regular field $B_{x}$, so that the regular transition proceeds adiabatically. The noise remains fast in the scale $\tau_{\mathrm{LZ}}$, but its relaxation frequency may be much larger than $B_{x}$. Section VII contains our conclusions.

\section{SPIN $S$ LANDAU-ZENER PROBLEM}

The Hamiltonian of a free spin $\mathbf{S}$ with the maximal projection $S$ in an external time-dependent magnetic field $\mathbf{B}(t)$ reads

$$
H_{S}(t)=-\mathbf{S b}(t),
$$

where $\mathbf{b}(t)=g \mu_{B} \mathbf{B}(t)$. The key observation ${ }^{13,14}$ is that this Hamiltonian is an operator of time-dependent infinitesimal rotation. Therefore the corresponding evolution matrix $U_{S}\left(t, t_{0}\right)=T \exp \left[-i \int_{t_{0}}^{t} H_{S}\left(t^{\prime}\right) d t^{\prime}\right]$ is an operator of rotation belonging to the group $\mathrm{SO}(3)$ acting in its irreducible representation labeled by an integer or half-integer $S$. Since the composition law is the same for any irreducible representation, the resulting evolution operator represents the same rotation for any spin. The group theory allows us to construct this matrix for an arbitrary spin if it is known for spin $1 / 2$ (see Ref. 17, Chap. VIII). The multispinor technique is most appropriate for this purpose. The spin $S$ state can be represented as a direct symmetrized product of $2 S$ spin $1 / 2$ states:

$$
\begin{aligned}
|S, m\rangle= & \sqrt{\frac{(S+m) !(S-m) !}{(2 S) !}}(|++\ldots+-\ldots-\rangle \\
& +|++\ldots-+\ldots-\rangle+\ldots),
\end{aligned}
$$

where each ket contains $S+m$ spins up (+) and $S-m$ spins down (-) and all permutations of up and down are performed. Let the $\mathrm{SU}(2)$ matrix rotating spin $1 / 2$ states be

$$
u=\left(\begin{array}{cc}
a & b \\
-b^{*} & a^{*}
\end{array}\right)
$$

with the constraint $|a|^{2}+|b|^{2}=1$ imposed. The transformation for the state (3) of the spin $S$ can be obtained as the direct product of transformations (4):

$$
\begin{aligned}
|S, m\rangle \rightarrow & \sqrt{\frac{(S+m) !(S-m) !}{(2 S) !}} a^{S+m}\left(-b^{*}\right)^{S-m}|S, S\rangle \\
& +\left(\frac{\sqrt{2 S}(2 S-1) !}{(S-m-1) !(S+m) !} a^{S+m}\left(-b^{*}\right)^{S-m-1} a^{*}\right. \\
& +\frac{\sqrt{2 S}(2 S-1) !}{(S+m-1) !(S-m) !} a^{S+m-1} \\
& \left.\times\left(-b^{*}\right)^{S-m} b\right)|S, S-1\rangle \ldots
\end{aligned}
$$

A general matrix element of the rotation operator $\left\langle m\left|U_{S}\right| m^{\prime}\right\rangle$ for the spin $S$ is expressed in terms of $a, b, a^{*}, b^{*}$ in the following way ${ }^{17,18}$ :

$$
\begin{aligned}
\left\langle m\left|U_{S}\right| m^{\prime}\right\rangle= & {\left[\frac{\left(S+m^{\prime}\right) !\left(S-m^{\prime}\right) !}{(S+m) !(S-m) !}\right]^{1 / 2} a^{m^{\prime}+m} b^{m^{\prime}-m} } \\
& \times P_{S-m^{\prime}}^{m^{\prime}-m, m^{\prime}+m}\left(2|a|^{2}-1\right),
\end{aligned}
$$

where $P_{n}^{a, b}(x)$ are the Jacobi polynomials. ${ }^{19}$ The matrix elements possess the following symmetry properties: $\left\langle-m\left|U_{S}\right|-m^{\prime}\right\rangle=(-1)^{|m|+\left|m^{\prime}\right|}\left\langle m\left|U_{S}\right| m^{\prime}\right\rangle^{*}$ and $\left|\left\langle m\left|U_{S}\right| m^{\prime}\right\rangle\right|=\left|\left\langle m^{\prime}\left|U_{S}\right| m\right\rangle\right|=\left|\left\langle-m\left|U_{S}\right|-m^{\prime}\right\rangle\right|$. Equation (6) displays oscillations of the matrix element when the argument $2|a|^{2}-1$ varies from -1 to +1 . These oscillations are associated with the oscillatory behavior of the Jacobi polynomial. For the number of nodes $N\left(m, m^{\prime}, S\right)$ of the matrix element $\left\langle m\left|U_{S}\right| m^{\prime}\right\rangle$ a simple equation is valid: $N\left(m, m^{\prime}, S\right)=S-\max \left(|m|,\left|m^{\prime}\right|\right)$. The central matrix element with $m=m^{\prime}=0$ for integer $S$ and $|m|=\left|m^{\prime}\right|=1 / 2$ for halfinteger $S$ has a maximal number of nodes equal to $S$ and $S$ $-1 / 2$, respectively.

Let us specify the problem considering only a narrow vicinity of the diabatic levels crossing point, which we accept for $t=0$. It is possible if the interval of time $\tau_{\mathrm{LZ}}$, during which the transitions presumably proceed is much less than the characteristic time of the field variation $t_{0}=\left|\dot{B}_{z} / \ddot{B}_{z}\right|$. The characteristic time of transition can be identified as $\tau_{\mathrm{LZ}}$ $=\left|B_{x} / \dot{B}_{z}\right|$. Thus, the requirement of short transition time can be rewritten as $B_{x} \ll\left(\dot{B}_{z}\right)^{2} /\left|\ddot{B}_{z}\right|$. If this requirement is satisfied, one can approximate with high accuracy the magnetic field by a linear function of time $B_{z}(t)=\dot{B}_{z} t$.

Landau and Zener ${ }^{5,6}$ have solved such a problem for spin $1 / 2$. In particular Zener has determined matrix elements $a, b$ in terms of the Weber function $D_{-i \gamma^{2}}\left(e^{i \pi / 4} \sqrt{\dot{\omega} t}\right)$ for an arbitrary moment of time. For simplicity we will focus on the values of these parameters for the transition from $t=-\infty$ to $t=\infty$. According to the Landau-Zener solution

$$
a=\exp \left(-\pi \gamma^{2}\right), \quad b=-\frac{\sqrt{2 \pi} \exp \left(-\frac{\pi \gamma^{2}}{2}+\frac{i \pi}{4}\right)}{\gamma \Gamma\left(-i \gamma^{2}\right)} .
$$

When $\gamma$ varies from 0 to $\infty$, the modulus $|a|$ changes from 1 to 0 and the argument of the Jacobi polynomial in Eq. (6) 
varies from 1 to -1 . Using expression (6), one finds the solution for an arbitrary spin $S$ in terms of coefficients $a$ and $b$, or equivalently, in terms of the Landau-Zener parameter $\gamma$. Thus, the transition amplitudes oscillate as function of $\gamma$.
The physical reason of these oscillations is the interference between different Feynman paths leading from $m$ to $m^{\prime}$. For illustration we show here corresponding matrices for spins 1 , $3 / 2$, and 2 :

$$
\begin{aligned}
& U_{1}=\left(\begin{array}{ccc}
a^{2} & -\sqrt{2} a b & -b^{2} \\
\sqrt{2} a b^{*} & 2|a|^{2}-1 & \sqrt{2} a^{*} b \\
-b^{* 2} & -\sqrt{2} a^{*} b^{*} & a^{* 2}
\end{array}\right), \\
& U_{3 / 2}=\left(\begin{array}{cccc}
a^{3} & \sqrt{3} a^{2} b & \sqrt{3} a b^{2} & b^{3} \\
-\sqrt{3} a^{2} b^{*} & \left(3|a|^{2}-2\right) a & \left(3|a|^{2}-1\right) b & \sqrt{3} a^{*} b^{2} \\
\sqrt{3} a b^{* 2} & -\left(3|a|^{2}-1\right) b^{*} & \left(3|a|^{2}-2\right) a^{*} & \sqrt{3} a^{* 2} b \\
-b^{* 3} & \sqrt{3} a^{*} b^{* 2} & -\sqrt{3} a^{* 2} b^{*} & a^{* 3}
\end{array}\right) .
\end{aligned}
$$

We present only a quarter of the transition matrix for $S=2$. The rest can be found by using above described symmetry properties.

$$
U_{2}=\left(\begin{array}{ccccc}
a^{4} & \sqrt{4} a^{3} b & \sqrt{6} a^{2} b^{2} & \sqrt{4} a b^{3} & b^{4} \\
& \left(4|a|^{2}-3\right) a^{2} & \sqrt{6}\left(2|a|^{2}-1\right) a b & \left(4|a|^{2}-1\right) b^{2} \\
& & 6|a|^{4}-6|a|^{2}+1 &
\end{array}\right) .
$$

\section{DENSITY MATRIX AND BLOCH TENSORS}

When the random magnetic field acts onto the spin, the system must be described by the density matrix $\hat{\rho}$. By definition it is a $(2 S+1) \times(2 S+1)$ Hermitian matrix with the trace equal to 1 . It satisfies the standard equation of motion:

$$
i \frac{d \hat{\rho}}{d t}=[H, \hat{\rho}]
$$

Any Hermitian matrix with the trace equal to 1 can be represented as a sum:

$$
\begin{aligned}
\hat{\rho}= & \frac{1}{2 S+1} I+\mathbf{g} \cdot \mathbf{S}+\frac{1}{2} g_{i k}\left(S_{i} S_{k}+S_{k} S_{i}-\frac{2}{3} \delta_{i k} S(S+1)\right) \\
& +\ldots \frac{1}{(2 S) !} g_{i_{1} i_{2} \ldots i_{2 S}} \\
& \times\left(S_{i_{1}} S_{i_{2}} \ldots S_{i_{2 S}}+\text { all permutations-all traces }\right) .
\end{aligned}
$$

If the Hamiltonian is the generator of the rotation (2), each term in Eq. (12) corresponds to an irreducible representation and evolves independently. We will call symmetric tensors $g_{i k}, g_{i k l} \ldots g_{i_{1} i_{2} \ldots i_{2 S}}$ the Bloch tensors in analogy with the Bloch vector $\mathbf{g}$ well known from Bloch theory of the nuclear spin motion. Any trace of such a tensor must be equal to zero. The Hamiltonian (2) generates following equations of motion for the Bloch tensors:

$$
\dot{\mathbf{g}}=-\mathbf{b} \times \mathbf{g}, \quad \dot{g}_{i k}=-\varepsilon_{i l m} b_{l} g_{m k}-\varepsilon_{k l m} b_{l} g_{i m}, \ldots
$$

All these equations are independent and have obvious integrals of motion:

$$
\mathbf{g}^{2}=\text { const }, \quad g_{i k}^{2}=\text { const }, \quad g_{i k l}^{2}=\text { const }, \quad \ldots .
$$

Thus, the density matrix of a spin $S$ in an external timedependent magnetic field has $2 S$ conserving values. It is convenient to represent the Bloch tensors by their complex component with definite projection to the $z$ axis. We will denote such components of a tensor of the rank $s$ as $g_{s, m}$. The corresponding tensor operators composed from the symmetrized products of $2 s$ components of the spin $S$ operators are denoted $T_{s, m}^{S}$. They can be constructed from the senior operator of this representation $T_{s, s}^{S}=2^{-s / 2} S_{+}^{S}$ with $S_{ \pm}=S_{x}$ $\pm i S_{y}$ by recurrent commutations with the operator $S_{-}$:

$$
T_{s, m}^{S}=-\frac{1}{\sqrt{(s+m+1)(s-m)}}\left[S_{-}, T_{s, m+1}^{S}\right] .
$$

The operators $T_{s, m}^{S}$ are polynomials of the standard spin operators $S_{ \pm}$and $S_{z}$. They are operator analogs of spherical harmonics. Several lowest such operators are presented in the Appendix. We show below relations between the Cartesian components of the tensor $g_{i_{1} \ldots i_{s}}$ and its components $g_{s, m}$ for several values of $s$ : 


$$
\begin{gathered}
g_{1, \pm 1}=\frac{1}{\sqrt{2}}\left(g_{x} \pm i g_{y}\right), \quad g_{1,0}=g_{z}, \\
g_{2, \pm 2}=\frac{1}{\sqrt{6}}\left(g_{x x}-g_{y y} \pm 2 i g_{x y}\right), \\
g_{2, \pm 1}=\frac{1}{\sqrt{2}}\left(g_{x z} \pm i g_{y z}\right), \quad g_{2,0}=g_{z z}, \\
g_{3, \pm 3}=\frac{1}{\sqrt{20}}\left(g_{x x x} \pm 3 i g_{x x y}-3 g_{x y y} \mp i g_{y y y}\right), \ldots .
\end{gathered}
$$

The general rule for writing the $s, \pm m$ component via its Cartesian counterparts is the same as for the product $(m ! / \sqrt{2 m !})(x \pm i y)^{m} z^{s-m}$. The Hamiltonian (2) in terms of components with definite projections reads (note that $b_{ \pm}$ $\left.=b_{x} \pm i b_{y} ; S_{ \pm}=S_{x} \pm i S_{y}\right)$

$$
H=-b_{z} S_{z}-\frac{1}{2}\left(b_{+} S_{-}+b_{-} S_{+}\right) .
$$

Equations (13) in terms of the components with definite $z$-projections read

$$
\begin{aligned}
\dot{g}_{s, m}= & -i m b_{z} g_{s, m}+\frac{i}{2} \sqrt{(s+m)(s-m+1)} b_{+} g_{s, m-1} \\
& +\frac{i}{2} \sqrt{(s-m)(s+m+1)} b_{-} g_{s, m+1}
\end{aligned}
$$

and the conservation laws are

$$
\sum_{m=-s}^{s}\left|g_{s, m}\right|^{2}=\text { const. }
$$

\section{FAST NOISE IN TWO-LEVEL SYSTEM}

In this section we consider only spin $1 / 2$ or, equivalently, a two-level system. We assume that the magnetic field can be separated into regular and random parts:

$$
\mathbf{b}(t)=\mathbf{b}_{r}(t)+\boldsymbol{\eta}(t),
$$

where $\mathbf{b}_{r}(t)=\hat{z} \dot{b}_{z} t+\hat{x} b_{x}$ and $\boldsymbol{\eta}(t)$ is the Gaussian noise determined by its correlators:

$$
\left\langle\eta_{i}(t) \eta_{k}\left(t^{\prime}\right)\right\rangle=f_{i k}\left(t-t^{\prime}\right) .
$$

We assume that the correlators (23) decay after a characteristic time difference $\tau_{n}$ and that this correlation time is much less than the characteristic time of the LZ process $\tau_{\mathrm{LZ}}$. However, the noise must be slow enough to avoid the direct transitions between the levels when the interlevel distance approaches its saturation or characteristic value $\omega$ far from the crossing point. Thus, the noise correlation time $\tau_{n}$ must satisfy the following inequalities:

$$
\omega^{-1} \ll \tau_{n} \ll \tau_{\mathrm{LZ}}
$$

The spectral width of noise is $1 / \tau_{n}$. The noise produces transitions during the interval of time $t_{a c c}=1 /\left(\dot{b}_{z} \tau_{n}\right)$, after which the current LZ frequency becomes larger than the noise spectral width. We will call this interval the accumulation time and assume that it is much larger than other characteristic time intervals $\tau_{n}$ and $\tau_{\mathrm{LZ}}$.

We first solve an auxiliary problem in which $b_{x}=0$ and transitions are mediated by noise only. Such a problem for a special shape of correlators $\left(f_{x x}=J^{2} \exp \left[-\left(t-t^{\prime}\right) / \tau_{n}\right]\right.$; the remaining components of the correlation tensor are zero) was solved earlier by Kayanuma ${ }^{15}$ and studied numerically by Nishino et al. ${ }^{23}$ In our work ${ }^{16}$ we have generalized and simplified the Kayanuma solution. Here we reproduce our solution $^{16}$ in a modified form convenient for extension to higher spins. We also obtain new results calculating the fluctuation of the density matrix, or equivalently the Bloch vector. Equations for the component of the Bloch vector in this case are

$$
\begin{gathered}
\dot{g}_{z}=(i / \sqrt{2})\left(\eta_{+} g_{-}-\eta_{-} g_{+}\right), \\
\dot{g}_{ \pm}=\mp i\left(\dot{b}_{z} t+\eta_{z}\right) g_{ \pm}+(i / \sqrt{2}) \eta_{ \pm} g_{z} .
\end{gathered}
$$

Solving equation for $g_{ \pm}$, we find

$$
\begin{aligned}
g_{ \pm}(t)= & g_{ \pm}(-\infty) \exp \left(\mp \frac{i \dot{b}_{z} t^{2}}{2} \mp i \int_{-\infty}^{t} \eta_{z}\left(t^{\prime}\right) d t^{\prime}\right) \\
& +(i / \sqrt{2}) \int_{-\infty}^{t} \exp \left[\mp \frac{i \dot{b}_{z}\left(t^{2}-t^{\prime 2}\right)}{2}\right. \\
& \left.\mp i \int_{t^{\prime}}^{t} \eta_{z}\left(t^{\prime \prime}\right) d t^{\prime \prime}\right] \eta_{ \pm}\left(t^{\prime}\right) g_{z}\left(t^{\prime}\right) d t^{\prime}
\end{aligned}
$$

Let us first consider the case of complete initial decoherence: $g_{ \pm}(-\infty)=0$. Then, plugging equation (27) into the first equation (25), we find a separate equation for $g_{z}$ :

$$
\begin{aligned}
\dot{g}_{z}= & -(1 / 2) \int_{-\infty}^{t} \exp \left[-\frac{i \dot{b}_{z}\left(t^{2}-t^{\prime 2}\right)}{2}-i \int_{t^{\prime}}^{t} \eta_{z}\left(t^{\prime \prime}\right) d t^{\prime \prime}\right] \\
& \times \eta_{+}(t) \eta_{-}\left(t^{\prime}\right) g_{z}\left(t^{\prime}\right) d t^{\prime}+\text { c.c. }
\end{aligned}
$$

Let us average Eq. (28) over the ensemble of the random noise. For such averaging it is important that the noise correlation time $\tau_{n}$ is much shorter than the time $t_{a c c}$ necessary for a substantial variation of $\left\langle g_{z}\right\rangle$. This fact allows us to represent the average

$$
\left\langle\eta_{+}(t) \eta_{-}\left(t^{\prime}\right) g_{z}\left(t^{\prime}\right)\right\rangle
$$

approximately as a product:

$$
\left\langle\eta_{+}(t) \eta_{-}\left(t^{\prime}\right) g_{z}\left(t^{\prime}\right)\right\rangle=\left\langle\eta_{+}(t) \eta_{-}\left(t^{\prime}\right)\right\rangle\left\langle g_{z}\left(t^{\prime}\right)\right\rangle .
$$

More accurately one should incorporate the fluctuations of $g_{z}$. In the leading approximation they are determined by the same equation (28) as follows: 


$$
\begin{aligned}
\delta g_{z}= & -(1 / 2) \int_{-\infty}^{t} d t_{1} \int_{-\infty}^{t_{1}} \exp \left[-\frac{i \dot{b}_{z}\left(t_{1}^{2}-t_{2}^{2}\right)}{2}\right] \\
& \times\left[\eta_{+}\left(t_{1}\right) \eta_{-}\left(t_{2}\right)-\left\langle\eta_{+}\left(t_{1}\right) \eta_{-}\left(t_{2}\right)\right\rangle\right] \\
& \times\left\langle g_{z}\left(t_{2}\right)\right\rangle d t_{2}+\text { c.c. }
\end{aligned}
$$

We ignore $\eta_{z}$ (this approximation will be justified by the next step). Let substitute this additional term into Eq. (28) and perform averaging over the Gaussian random field $\boldsymbol{\eta}$. According to the Wick's rule, it is reduced to all possible pairings. In our case the only possible pairing is $\left\langle\eta_{+}(t) \eta_{-}\left(t_{2}\right)\right\rangle\left\langle\eta_{-}\left(t^{\prime}\right) \eta_{+}\left(t_{1}\right)\right\rangle$. Such a pairing limits the integration by the interval $t-\tau_{n}<t_{2}<t_{1}<t^{\prime}<t$. Thus the contribution of the fluctuational term differs by an additional factor $\sim \tau_{n} / t_{a c c} \ll 1$ from the principal contribution from $\left\langle g_{z}\right\rangle$. These arguments represent a shortened version of the original arguments by Kayanuma ${ }^{15}$ and are akin to the Abrikosov-Gor'kov theory of static disordered alloys. ${ }^{20}$

Using the fact that the decay of the correlator $\left\langle\eta_{+}(t) \eta_{-}\left(t^{\prime}\right)\right\rangle$ limits effectively the integration over time by an interval $t-\tau_{n}<t^{\prime}<t$, we can prove that the contribution of the noise component $\eta_{z}$ in the exponent in Eq. (28) can be neglected. To estimate this contribution we assume that $\eta_{z}$ is statistically independent from other components. Then the averaging over $\eta_{z}$ results in the Debye-Waller factor $\exp \left\{-\frac{1}{2}\left\langle\left[\int_{t^{\prime}}^{t} \eta_{z}\left(t^{\prime \prime}\right) d t^{\prime \prime}\right]^{2}\right\rangle\right\}$. The argument of this exponent can be estimated as $\left\langle\eta_{z}^{2}\right\rangle \tau_{n}^{2}$. It is small provided the level of noise $\left\langle\eta_{z}^{2}\right\rangle$ is much smaller than $\tau_{n}^{-2}$. The noise correlators are even functions of the time difference. Therefore, expanding linearly the time argument of the exponent in the same equation $\left[\dot{b}_{z}\left(t^{2}-t^{\prime 2}\right)\right] / 2 \approx \dot{b}_{z} t\left(t-t^{\prime}\right)$, one can transform the integral-differential equation (28) into an ordinary differential equation for $\left\langle g_{z}\right\rangle$ :

$$
\left\langle\dot{g}_{z}\right\rangle=-\hat{F}\left(\dot{b}_{z} t\right)\left\langle g_{z}\right\rangle,
$$

where $\hat{F}(\Omega)$ is the Fourier-transform of the function $F(\tau)$ $=f_{x x}(\tau)+f_{y y}(\tau)$ :

$$
\hat{F}(\Omega)=\int_{-\infty}^{\infty} F(\tau) \cos \Omega \tau d \tau .
$$

Equation (30) has a simple solution:

$$
\left\langle g_{z}(t)\right\rangle=\left\langle g_{z}(-\infty)\right\rangle \exp \left[-\int_{-\infty}^{t} \hat{F}\left(\dot{b}_{z} t^{\prime}\right) d t^{\prime}\right] .
$$

At $t \rightarrow+\infty$ the asymptotic value of $\left\langle g_{z}\right\rangle$ is

$$
\left\langle g_{z}(+\infty)\right\rangle=\left\langle g_{z}(-\infty)\right\rangle \exp (-\theta), \quad \theta=\frac{\pi F(0)}{\dot{b}_{z}} .
$$

Note that what matters for the LZ transition is the average quadratic fluctuation of nondiagonal noise at any moment $F(0)=\left\langle\eta_{x}^{2}+\eta_{y}^{2}\right\rangle$, in contrast to a standard characteristic of the white noise which would be $\hat{F}(0)$. Indeed, commonly white noise correlator is introduced as $\left\langle\eta(t) \eta\left(t^{\prime}\right)\right\rangle=\gamma \delta(t$ $\left.-t^{\prime}\right)$. The only characteristic of the noise in this approach is $\gamma=\int_{-\infty}^{\infty}\left\langle\eta(t) \eta\left(t^{\prime}\right)\right\rangle d t^{\prime}$. An interesting feature of the asymptotic formula (33) is its independence on the noise correlation time $\tau_{n}$. However, it should be kept in mind that this asymptotic is valid only at time $t \gg t_{a c c}=\left(\dot{b}_{z} \tau_{n}\right)^{-1}$.

Returning to the solution (27) for $g_{ \pm}$, we see that the term

$$
g_{ \pm}(-\infty) \exp \left(\mp \frac{i \dot{b}_{z} t^{2}}{2} \mp i \int_{-\infty}^{t} \eta_{z}\left(t^{\prime}\right) d t^{\prime}\right)
$$

omitted at substitution in the first equation (25) and the ensemble averaging, vanishes if the $z$ component of the noise is statistically independent from others. Now let us perform a similar procedure solving first the equation for $g_{z}$ and then substituting the solution into the equations for $g_{ \pm}$. In the same approximation equation for averages of these components of the Bloch vector reads

$$
\left\langle\dot{g}_{ \pm}\right\rangle=-\frac{1}{2} \hat{F}\left(\dot{b}_{z} t\right)\left\langle g_{ \pm}\right\rangle \text {. }
$$

The first term in Eq. (27) after the averaging turns into zero at any finite $t$. Indeed the Debye-Waller factor which appears in this case is $\exp \left\{-\frac{1}{2}\left\langle\left[\int_{t}^{\infty} \eta_{z}\left(t^{\prime \prime}\right) d t^{\prime \prime}\right]^{2}\right\rangle\right\}=0$. The asymptotics of $\left\langle g_{ \pm}\right\rangle$at $t \rightarrow+\infty$ is

$$
\left\langle g_{ \pm}(+\infty)\right\rangle=\exp \left(-\frac{\theta}{2}\right)\left\langle g_{ \pm}(-\infty)\right\rangle, \quad \theta=\frac{\pi F(0)}{\dot{b}_{z}}
$$

Note that the symbols $g_{ \pm}( \pm \infty)$ denote the coefficients at $\exp \left(\mp i \dot{b}_{z} t^{2} / 2\right)$. Thus, the noise asymptotically tends to reduce the average components of the Bloch vector, i.e., to establish equipopulation of the levels and to destroy the coherence. However, during the time interval of the order of $t_{\mathrm{acc}}$ the average Bloch vector can oscillate.

For the considered problem it is possible to find exactly the fluctuations of the Bloch vector. Indeed, they are given by a standard formula:

$$
\left\langle(\delta \mathbf{g})^{2}\right\rangle=\left\langle\mathbf{g}^{2}\right\rangle-\langle\mathbf{g}\rangle^{2} .
$$

Due to the symmetry of the problem, $\mathbf{g}^{2}$ is a conserving value. Therefore, its average coincides with itself and is determined by initial conditions. The average value of the vector $\langle\mathbf{g}\rangle$ was calculated above. Thus, we find an asymptotic value of the fluctuations:

$$
\begin{aligned}
\left.\left\langle(\delta \mathbf{g})^{2}\right\rangle\right|_{t=+\infty}= & g_{z}^{2}(-\infty)[1-\exp (-2 \theta)]+\left[g_{x}^{2}(-\infty)\right. \\
& \left.+g_{y}^{2}(-\infty)\right][1-\exp (-\theta)] .
\end{aligned}
$$

The values of average square fluctuation can be also written for any moment of time. The fluctuations are strong, i.e., their magnitudes are of the same order as the average values of the Bloch vector components unless $\theta$ is very small. An important property of the noise is that in fluctuations it mixes diagonal and nondiagonal elements of the density matrix, i.e., all three components of the Bloch vector. 
Let us proceed to the solution of a more general problem retaining the nonzero $x$ component of the regular field $b_{x}$. Such a solution becomes possible due to the separation of times ${ }^{16}$ : we can neglect the effect of $b_{x}$ beyond the LZ time interval $\tau_{\mathrm{LZ}}$ near the crossing point. On the other hand, we can neglect the effect of the noise inside this and even much larger interval of time, since its characteristic time is $t_{a c c}$ $\gg \tau_{\mathrm{LZ}}$. Thus, the problem is separated into three parts: in two intervals $\left(-\infty,-t_{0}\right)$ and $\left(t_{0},+\infty\right)$ we can use the solution of our auxiliary problem, i.e., to take in account only transitions caused by the noise; in the interval $\left(-t_{0}, t_{0}\right)$ the LandauZener solution is valid. We need only to match them properly. The time interval $t_{0}$ must satisfy a strong inequality: $\tau_{\mathrm{LZ}} \ll t_{0} \ll t_{a c c}$. As shown before, the average components $g_{z}$ and $g_{ \pm}$evolve separately under the action of the noise. Therefore, at the moment of time $-t_{0}$ these components are

$$
\begin{gathered}
\left\langle g_{z}\left(-t_{0}\right)\right\rangle=\exp (-\theta / 2) g_{z}(-\infty), \\
\left\langle g_{ \pm}\left(-t_{0}\right)\right\rangle=\exp (-\theta / 4) g_{ \pm}(-\infty) .
\end{gathered}
$$

The action of the LZ transition matrix (4) with the matrix elements (7) can be transferred onto the vector $\mathbf{g}$ by using spin-1 matrix (8). Thus, the average components of the Bloch vector at $t=t_{0}$ are as follows:

$$
\begin{aligned}
\left\langle g_{z}\left(t_{0}\right)\right\rangle= & \sqrt{2} a b^{*}\left\langle g_{+}\left(-t_{0}\right)\right\rangle+\left(2|a|^{2}-1\right)\left\langle g_{z}\left(-t_{0}\right)\right\rangle \\
& +\sqrt{2} a^{*} b\left\langle g_{-}\left(-t_{0}\right)\right\rangle, \\
\left\langle g_{+}\left(t_{0}\right)\right\rangle=a^{2}\left\langle g_{+}\left(-t_{0}\right)\right\rangle-\sqrt{2} a b\left\langle g_{z}\left(-t_{0}\right)\right\rangle-b^{2}\left\langle g_{-}\left(-t_{0}\right)\right\rangle, & \\
\left\langle g_{-}\left(t_{0}\right)\right\rangle= & -b^{* 2}\left\langle g_{+}\left(-t_{0}\right)\right\rangle-\sqrt{2} a^{*} b^{*}\left\langle g_{z}\left(-t_{0}\right)\right\rangle \\
& +a^{* 2}\left\langle g_{-}\left(-t_{0}\right)\right\rangle,
\end{aligned}
$$

where $a$ and $b$ are given by Eqs. (7). The transition from $+t_{0}$ to $+\infty$ is provided by the same diagonal transition matrix (38), which was already used for the transition from $-\infty$ to $-t_{0}$ :

$$
\begin{gathered}
\left\langle g_{z}(+\infty)\right\rangle=\exp (-\theta / 2) g_{z}\left(+t_{0}\right), \\
\left\langle g_{ \pm}(+\infty)\right\rangle=\exp (-\theta / 4) g_{ \pm}\left(+t_{0}\right) .
\end{gathered}
$$

Collecting together Eqs. (38)-(42), we find the final result:

$$
\begin{aligned}
\left\langle g_{z}(+\infty)\right\rangle= & \sqrt{2} \exp (-3 \theta / 4)\left[a b^{*} g_{+}(-\infty)+a^{*} b g_{-}(-\infty)\right] \\
+ & \exp (-\theta)\left(2|a|^{2}-1\right) g_{z}(-\infty), \\
\left\langle g_{+}(+\infty)\right\rangle= & \exp (-\theta / 2)\left[a^{2} g_{+}(-\infty)-b^{2} g_{-}(-\infty)\right] \\
& -\exp (-3 \theta / 4) \sqrt{2} a b g_{z}(-\infty), \\
\left\langle g_{-}(+\infty)\right\rangle= & \exp (-\theta / 2)\left[-b^{* 2} g_{+}(-\infty)+a^{* 2} g_{-}(-\infty)\right] \\
- & \exp (-3 \theta / 4) \sqrt{2} a^{*} b^{*} g_{z}(-\infty) .
\end{aligned}
$$

Let us analyze first the case of complete decoherence at $t$ $=-\infty$, i.e., $g_{ \pm}(-\infty)=0$. Then Eqs. (43) - (45) look much simpler:

$$
\begin{gathered}
\left\langle g_{z}(+\infty)\right\rangle=\exp (-\theta)\left(2|a|^{2}-1\right) g_{z}(-\infty), \\
\left\langle g_{+}(+\infty)\right\rangle=-\exp (-3 \theta / 4) \sqrt{2} a b g_{z}(-\infty) .
\end{gathered}
$$

Equation (46) shows that, in the absence of initial coherence, the population difference can only decrease after the transition. The noise only strengthens this tendency. However, the initial coherence, if it exists, can increase the difference of population to the value larger than initial. Equation (47) demonstrates an inverse process: the coherence can appear after the transition even if it was absent in the initial state. It is straightforward to derive the transition probability from Eq. (46):

$$
\begin{aligned}
P_{1 \rightarrow 2} & =\frac{1}{2}\left[1-\exp (-\theta)\left(2|a|^{2}-1\right)\right] \\
& =\frac{1}{2}\left[1+\exp (-\theta)-2 \exp \left(-\theta-2 \pi \gamma^{2}\right)\right] .
\end{aligned}
$$

This formula was obtained in our previous work. ${ }^{16}$ At zero noise intensity $(\theta=0)$ this result turns into the Landau-Zener transition probability. At very big noise $(\theta=\infty)$ the probability is equal to $1 / 2$. The probability is the same for transitions $1 \rightarrow 2$ and $2 \rightarrow 1$. This symmetry does not look strange keeping in mind that we considered the classical noise, which produces the induced transitions. It vanishes as soon as the quantum nature of the noise and spontaneous transitions are taken into account.

For completeness we present a formula for quadratic fluctuations at $t=\infty$ in the case of complete initial decoherence:

$$
\begin{aligned}
&\left\langle\mathbf{g}^{2}(+\infty)\right\rangle-\langle\mathbf{g}(+\infty)\rangle^{2} \\
&=\mid\left|g_{z}(-\infty)\right|^{2}\left[1-e^{-2 \theta}-4\left(e^{-2 \pi \gamma^{2}}-e^{-4 \pi \gamma^{2}}\right)\right. \\
&\left.\times\left(e^{-3 \theta / 2}-e^{-2 \theta}\right)\right] .
\end{aligned}
$$

The fluctuations vanish at $\theta=0$ and reach their maximum value equal to $\left|g_{z}(-\infty)\right|^{2}$ at $\theta=\infty$.

\section{FAST NOISE AT A MULTILEVEL CROSSING}

We consider only the case of a Zeeman multiplet placed into a varying magnetic field. It is described by Eqs. (20). Averaging them over the fast noise is performed by two steps, as was done in the preceding section. First we neglect the transitions produced by the regular part of the magnetic field and take in account only the transitions produced by the random field. This approach is correct outside the time interval $\tau_{\mathrm{LZ}}$ near the avoided crossing point $t=0$. Assuming for simplification complete initial decoherence, we find for the average diagonal matrix elements following equations:

$$
\left\langle g_{s, 0}^{S}(+\infty)\right\rangle=\exp \left[-\frac{s(s+1)}{2} \theta\right]\left\langle g_{s, 0}^{S}(-\infty)\right\rangle
$$


Note that these relations do not contain $S$ explicitly, the time evolution depends on $s$ only. In the course of derivation of Eq. (50) we ignored not only the fluctuations of $g_{s, 0}^{S}$, but also all higher components except for $g_{s,+1}^{S}$. Their contributions to the main component $g_{s, 0}^{S}$ have the same order of magnitude $\sim \tau_{n} / t_{a c c}$ as the contribution of fluctuations.

The generalization of Eq. (50) to the higher projections $m$, i.e., to the coherence factors requires some care. The truncated system of Eqs. (20) for zero initial values of all components of the $s$-tensor except of $g_{s, m}^{S}$, reads

$$
\begin{gathered}
\dot{g}_{s, m}^{S}=-i m \dot{b}_{z} t_{s, m}^{S}+i\left(\lambda_{s, m} \eta_{+} g_{s, m-1}^{S}+\lambda_{s,-m} \eta_{-} g_{s, m+1}^{S}\right), \\
\dot{g}_{s, m-1}^{S}=-i(m-1) \dot{b}_{z} t g_{s, m-1}^{S}+i \lambda_{s, m} \eta_{-} g_{s, m}^{S} \\
\dot{g}_{s, m+1}^{S}=-i(m+1) \dot{b}_{z} t g_{s, m+1}^{S}+i \lambda_{s,-m} \eta_{+} g_{s, m}^{S}
\end{gathered}
$$

where $\lambda_{s, m}=\sqrt{(s-m)(s+m+1)}$. To deal with slowly varying average values, the fast oscillating exponent should be eliminated. In order to do that we introduce slow variables $\tilde{g}_{s, m}^{S}=g_{s, m}^{S} \exp \left[\left(i \dot{b}_{z} t^{2} / 2\right) m\right]$. Further we consider only slow amplitudes $\tilde{g}_{s, m}$ and omit the sign tilde. After elimination of the values $g_{s, m \pm 1}^{S}$ and averaging, we find a following equation for $g_{s, m}^{S}$ :

$$
\left\langle\dot{g}_{s, m}^{S}\right\rangle=-\frac{1}{2}\left\{\left[s(s+1)-m^{2}\right] \hat{F}\left(\dot{b}_{z} t\right)+m \hat{G}\left(\dot{b}_{z} t\right)\right\}\left\langle g_{s, m}^{S}\right\rangle,
$$

where $\hat{F}(\Omega)$ is defined by Eq. (31) and $\hat{G}(\Omega)$ is defined as a sine Fourier transform:

$$
\hat{G}(\Omega)=\int_{-\infty}^{\infty}\left\langle\eta_{x}(\tau) \eta_{y}(0)-\eta_{y}(\tau) \eta_{x}(0)\right\rangle \sin \Omega \tau d \tau .
$$

Thus, the time dependence of the average $\left\langle g_{s, m}^{S}\right\rangle$ is defined as

$$
\begin{aligned}
\left\langle g_{s, m}^{S}(t)\right\rangle= & \exp \left\{-\frac{1}{2} \int_{-\infty}^{t}\left\{\left[s(s+1)-m^{2}\right] \hat{F}\left(\dot{b}_{z} t^{\prime}\right)\right.\right. \\
& \left.\left.+m \hat{G}\left(\dot{b}_{z} t^{\prime}\right)\right\} d t^{\prime}\right\}\left\langle g_{s, m}^{S}(-\infty)\right\rangle .
\end{aligned}
$$

Its asymptotic value at $t \rightarrow+\infty$ does not contain the sine Fourier transform:

$$
\left\langle g_{s, m}^{S}(+\infty)\right\rangle=\exp \left[-\frac{1}{2}\left[s(s+1)-m^{2}\right] \theta\right]\left\langle g_{s, m}^{S}(-\infty)\right\rangle .
$$

At $s=1, m=1$ this result coincides with Eq. (34).

Now we proceed to our main problem including both regular and random transverse magnetic fields. We will employ the separation of their action in the time proven in the preceding section. To avoid lengthy formulas our consideration will be restricted to the case of complete initial decoherence. Then the only nonzero components of the Bloch tensors are $g_{s, 0}^{S}$. Their evolution is described by three independent factors, two of them originating from the noise and the central factor being the generalized Landau-Zener-Hioe matrix element:

$$
\begin{aligned}
\left\langle g_{s, 0}^{S}(+\infty)\right\rangle= & \exp \left[-\frac{s(s+1)}{2} \theta\right] P_{s}^{0,0}\left(2 e^{-2 \pi \gamma^{2}}-1\right) g_{s, 0}^{S} \\
& (-\infty)
\end{aligned}
$$

where $P_{s}^{0,0}(x)$ is the Jacobi polynomial. The average values of the Bloch tensors components with $m \neq 0$ vanish as a result of averaging over the random phases in the initial state. To find the transition probabilities $P_{j \rightarrow j^{\prime}}$ it is necessary to put all the diagonal elements of the density matrix except of $\rho_{j j}$ equal to zero in the initial state:

$$
\frac{1}{2 S+1}+\sum_{s=1}^{2 S} g_{s, 0}^{S}(-\infty)\left(T_{s, 0}^{S}\right)_{k, k}=\delta_{j k},
$$

and find from these equations the initial values $g_{s, 0}^{S}(-\infty)$. Then the transition probabilities are

$$
P_{j \rightarrow j^{\prime}}=\frac{\delta_{j j^{\prime}}}{2 S+1}+\sum_{s=1}^{2 S} g_{s, 0}^{S}(+\infty)\left(T_{s, 0}^{S}\right)_{j, j^{\prime}} \exp \left(-\frac{s(s+1)}{2} \theta\right),
$$

where $g_{s, 0}^{S}(+\infty)$ are defined by Eq. (58). In the following tables we demonstrate the results of calculations according to this algorithm for the values of $S 1,3 / 2$, and 2 [the results for $S=1 / 2$ are given in the preceding section by Eq. (48)],

$$
\begin{array}{lccr}
j^{\prime} \backslash^{j} & +1 & 0 & -1 \\
+1 & \frac{1}{3}+\frac{1}{2} E_{1}+\frac{1}{6} E_{2} & \\
0 & \frac{1}{3}-\frac{1}{3} E_{2} & \frac{1}{3}+\frac{2}{3} E_{2} \\
-1 & \frac{1}{3}-\frac{1}{2} E_{1}+\frac{1}{6} E_{2},
\end{array}
$$




$$
\begin{aligned}
& j_{j^{\prime}} \searrow^{j} \quad 3 / 2 \quad 1 / 2 \quad-1 / 2 \quad-3 / 2 \\
& 3 / 2 \quad \frac{1}{4}+\frac{9 E_{1}}{20}+\frac{E_{2}}{4}+\frac{E_{3}}{20} \\
& 1 / 2 \frac{1}{4}+\frac{3 E_{1}}{20}-\frac{E_{2}}{4}-\frac{3 E_{3}}{20} \frac{1}{4}+\frac{E_{1}}{20}+\frac{E_{2}}{20}+\frac{9 E_{3}}{20} \\
& -1 / 2 \frac{1}{4}-\frac{3 E_{1}}{20}-\frac{E_{2}}{4}+\frac{3 E_{3}}{20} \frac{1}{4}-\frac{E_{1}}{20}+\frac{E_{2}}{20}-\frac{9 E_{3}}{20} \\
& -3 / 2 \frac{1}{4}-\frac{9 E_{1}}{20}+\frac{E_{2}}{4}-\frac{E_{3}}{20}, \\
& { }^{j^{\prime} \backslash^{j}} \frac{1}{5}+\frac{2 E_{1}}{5}+\frac{2 E_{2}}{7}+\frac{E_{3}}{10}+\frac{E_{4}}{70} \\
& 1 \frac{1}{5}+\frac{E_{1}}{5}-\frac{E_{2}}{7}-\frac{E_{3}}{5}-\frac{2 E_{4}}{35} \quad \frac{1}{5}+\frac{E_{1}}{10}+\frac{E_{2}}{14}+\frac{2 E_{3}}{5}+\frac{8 E_{4}}{35} \\
& 0 \quad \frac{1}{5}-\frac{2 E_{2}}{7}+\frac{3 E_{4}}{35} \quad \frac{1}{5}+\frac{E_{2}}{7}-\frac{12 E_{4}}{35} \quad \frac{1}{5}+\frac{2 E_{2}}{7}+\frac{18 E_{4}}{35} \\
& -1 \frac{1}{5}-\frac{E_{1}}{5}-\frac{E_{2}}{7}+\frac{E_{3}}{5}-\frac{2 E_{4}}{35} \quad \frac{1}{5}-\frac{E_{1}}{10}+\frac{E_{2}}{14}-\frac{2 E_{3}}{5}+\frac{8 E_{4}}{35} \\
& -2 \frac{1}{5}+\frac{2 E_{1}}{5}+\frac{2 E_{2}}{7}+\frac{E_{3}}{10}+\frac{E_{4}}{70} \text {. }
\end{aligned}
$$

Here we denoted $E_{s}=\exp \left[-\frac{1}{2} s(s+1) \theta\right]$, i.e., $E_{1}=e^{-\theta}, E_{2}$ $=e^{-3 \theta}, E_{3}=e^{-6 \theta}, E_{4}=e^{-10 \theta}$. Unfilled sites in the table can be easily restored using the time reversal symmetry: $P_{j \rightarrow j^{\prime}}=P_{j^{\prime} \rightarrow j}=P_{-j \rightarrow-j^{\prime}}$.

In Fig. 1 we compare the formula (61) with the numerical solution of the Schödinger equation for the spin $S=1$ in the absence of regular transverse component of the magnetic field, which convincingly confirms our analytical results.

The quadratic fluctuations of the values $g_{s, m}^{S}$ for fixed $S$ and $s$ are calculated as it was done in the previous section:

$$
\left\langle\delta \sum_{m=-s}^{s}\left|g_{s, m}^{S}\right|^{2}\right\rangle=\left.\sum_{m=-s}^{s}\left|g_{s, m}^{S}\right|^{2}\right|_{t=-\infty}-\left\langle g_{s, m}^{S}\right\rangle^{2} .
$$

In the case of complete initial decoherence employing Eq. (58), we find

$$
\begin{aligned}
\langle\delta & \left.\sum_{m=-s}^{s}\left|g_{s, m}^{S}\right|^{2}\right\rangle_{t=+\infty} \\
& =\left[g_{s, 0}^{S}(-\infty)\right]^{2}\left\{1-E_{s}^{2}\left[P_{s}^{0,0}\left(2 e^{-2 \pi \gamma^{2}}-1\right)\right]^{2}\right\} .
\end{aligned}
$$

\section{NOISE AT ADIABATIC LEVEL CROSSING}

In this section we consider a situation of adiabatically changing levels, i.e., $\dot{b}_{z} \ll b_{x}^{2}$, but still the noise is supposed to be sufficiently fast $\tau_{n} \ll\left(\dot{b}_{z}\right)^{-1 / 2}$. The adiabatic LandauZener transitions in coupled two-level systems have been proposed as a candidate for implementation of quantum gates in quantum computing. ${ }^{21}$ It is important to understand the influence of noise on such a $q$-bit manipulations in order to control the error propagation in quantum gate circuit. We do not specify the relationship between $b_{x}$ and $\tau_{n}$. This study is also motivated by experiments with nanomagnets ${ }^{7,22}$ in which inequality $\sqrt{\dot{b}_{z}} \tau_{n} \ll 1$ can be easily realized since the sweeping rate of the applied magnetic field can be made arbitrarily small. However, the noise may be not fast enough to compete with the tunnelling amplitude $b_{x}$. The nuclear bath correlation time is in the range of $\tau_{n} \sim 1 \mathrm{~ms}$, whereas the measured values of the tunnelling amplitude for known nanomagnets range between $10^{-10}$ and $10^{-3} \mathrm{~K}$, or equivalently 10 and $10^{8} \mathrm{~Hz}$. In a part of this interval $b_{x} \tau_{n}$ has a rather large value.

We consider in this section only 2-level systems. A natural approach to this problem is the transfer to the adiabatic set of states, i.e., to the eigenstates of the instantaneous regular part of the Hamiltonian (19). Let denote this time-dependent eigenvectors as

$$
a(t)=\left(\begin{array}{l}
a_{1}(t) \\
a_{2}(t)
\end{array}\right)
$$

and 


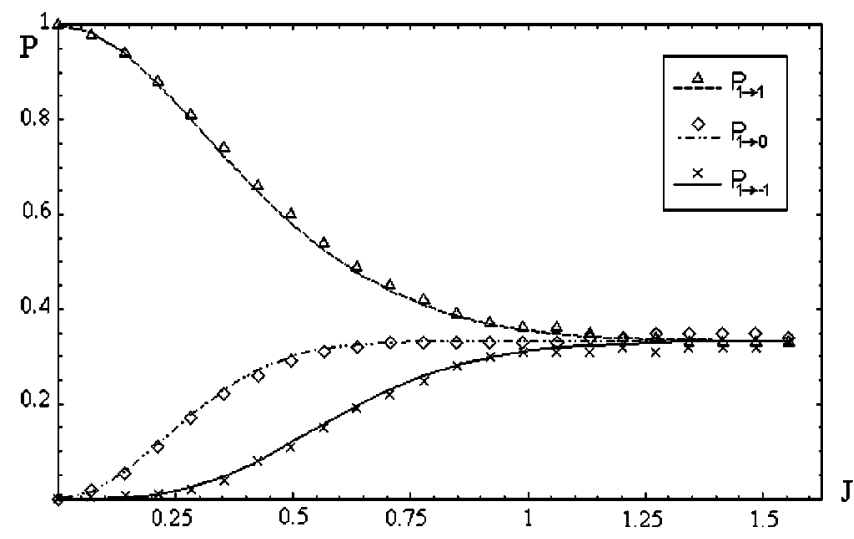

FIG. 1 . The final probability to find a spin- 1 system in the state with $S_{z}=-1,0$ or 1 when the initial state is $S_{z}=1$ as a function of the noise amplitude. The regular transverse field is set to zero. The Hamiltonian is $H=t S_{z}+\eta_{x} S_{x} \quad$ where $\left\langle\eta_{x}\left(t_{1}\right) \eta_{x}\left(t_{2}\right)\right\rangle$ $=J^{2} e^{-\lambda\left|t_{1}-t_{2}\right|}$. Discrete points correspond to results of numerical simulations with averaging over 200 different noise realizations and $\lambda=125$. Lines correspond to analytical predictions of Eq. (61).

$$
b(t)=\left(\begin{array}{l}
-a_{2}(t) \\
a_{1}(t)
\end{array}\right)
$$

where

$$
a_{1}(t)=\sqrt{\frac{\varepsilon(t)+b_{x}}{2 \varepsilon}}, a_{2}(t)=\sqrt{\frac{\varepsilon(t)-b_{x}}{2 \varepsilon}}
$$

and

$$
\varepsilon(t)=\sqrt{b_{z}^{2}+b_{x}^{2}}\left(b_{z}=\dot{b}_{z} t\right) .
$$

The unitary matrix of transformation to the adiabatic set reads

$$
U(t)=\left(\begin{array}{ll}
a_{1}(t) & a_{2}(t) \\
-a_{2}(t) & a_{1}(t)
\end{array}\right)=a_{1}(t) I-i a_{2}(t) \sigma_{y},
$$

where $\sigma_{\alpha}(\alpha=x, y, z)$ are Pauli matrices. In the new basis the total Hamiltonian acquires the following form:

$$
H=\varepsilon(t) \sigma_{z}+U \eta \sigma U^{-1}=\varepsilon(t) \sigma_{z}+\eta^{\prime} \sigma .
$$

The transformation of the random field components is

$$
\begin{gathered}
\eta_{x}^{\prime}=\left(a_{1}^{2}-a_{2}^{2}\right) \eta_{x}+2 a_{1} a_{2} \eta_{z}=\frac{b_{z}}{\varepsilon} \eta_{x}+\frac{b_{x}}{\varepsilon} \eta_{z} ; \\
\eta_{y}^{\prime}=\eta_{y} ; \quad \eta_{z}^{\prime}=-\frac{b_{x}}{\varepsilon} \eta_{x}+\frac{b_{z}}{\varepsilon} \eta_{z} .
\end{gathered}
$$

In this form the Hamiltonian (67) essentially coincides with the Hamiltonian of the auxiliary problem (zero regular transverse field) for the two-level system (see Sec. IV). The essential difference is, first, that the effective regular external field is not linear in time; instead it is equal to $\varepsilon(t)$; second, the correlators of effective noise $\eta^{\prime}$ now depend not only on the time difference, but also on time itself due to the timedependent transformation (68):

$$
\begin{aligned}
\left\langle\eta_{x}^{\prime}(t) \eta_{x}^{\prime}\left(t^{\prime}\right)\right\rangle= & \frac{1}{\varepsilon(t) \varepsilon\left(t^{\prime}\right)}\left[\dot{b}_{z}^{2} t t^{\prime}\left\langle\eta_{x}(t) \eta_{x}\left(t^{\prime}\right)\right\rangle\right. \\
& +b_{x}^{2}\left\langle\eta_{z}(t) \eta_{z}\left(t^{\prime}\right)\right\rangle \\
& +\dot{b}_{z} b_{x} t\left\langle\eta_{x}(t) \eta_{z}\left(t^{\prime}\right)\right\rangle+\dot{b}_{z} b_{x} t^{\prime} \\
& \left.\times\left\langle\eta_{z}(t) \eta_{x}\left(t^{\prime}\right)\right\rangle\right] .
\end{aligned}
$$

Still the noise correlation time is small in comparison to the characteristic time of variation for the adiabatic energy $\varepsilon(t)$. Employing the same approximation as in Sec. IV, we arrive at a similar equation of motion for average in the case of complete initial decoherence:

$$
\left\langle\dot{g}_{z}^{\prime}(t)\right\rangle=-F^{\prime}(t)\left\langle g_{z}^{\prime}(t)\right\rangle
$$

where

$$
\begin{aligned}
F^{\prime}(t)= & \hat{f}_{y y}(\varepsilon(t))+\frac{1}{\varepsilon^{2}(t)}\left\{\dot{b}_{z}^{2} t^{2} \hat{f}_{x x}(\varepsilon(t))+b_{x}^{2} \hat{f}_{z z}(\varepsilon(t))\right. \\
& \left.+\dot{b}_{z} b_{x} t\left[\hat{f}_{x z}(\varepsilon(t))+\hat{f}_{z x}(\varepsilon(t))\right]\right\}
\end{aligned}
$$

In the last equation the carets symbolize Fourier transforms of corresponding correlators. As before, we can find the average value $\left\langle g_{z}(t)\right\rangle$ at arbitrary moment of time. Asymptotically at $t \rightarrow+\infty$ we find

$$
\left\langle g_{z}^{\prime}(+\infty)\right\rangle=\exp \left[-\int_{-\infty}^{\infty} \frac{\dot{b}_{z}^{2} t^{2} \hat{f}_{x x}(\varepsilon(t))+\varepsilon^{2}(t) \hat{f}_{y y}(\varepsilon(t))+b_{x}^{2} \hat{f}_{z z}(\varepsilon(t))}{\varepsilon^{2}(t)} d t\right]\left\langle g_{z}^{\prime}(-\infty)\right\rangle
$$

The characteristic time after which the correlators in Eq. (74) become very small and decay rapidly is determined by the approximate equation $\varepsilon(t) \tau_{n} \approx 1$. If $\tau_{n} \ll b_{x}^{-1}$; then this characteristic time coincides with the accumulation time $t_{a c c}$ $=\left(\dot{b}_{z} \tau_{n}\right)^{-1}$ defined in Sec. IV, terms proportional to $b_{x}$ are negligibly small and we return to the result (43) with $|a|$
$=1$, or equivalently to Eq. (33) of the Sec. IV. In the opposite case $\tau_{n} \gg b_{x}^{-1}$ the value $\varepsilon(t)$ exceeds $\tau_{n}^{-1}$ at any moment of time $t$. Therefore, all correlators are small and the value of exponent in Eq. (74) is close to 1. It means that practically no transition proceeds due to the noise between adiabatic states. Thus, Eq. (74) carries the most interesting information 
when $\tau_{n} \sim b_{x}^{-1}$. An interesting feature of the transition probability is that the $z$ component of noise can produce a transition between adiabatic states. This happens because the latter rotate with time. Note that the $z$ component of noise is irrelevant if $\tau_{n} \ll b_{x}^{-1}$.

\section{CONCLUSIONS}

Motivated by synthesis and magnetic measurements of cubic nanomagnets, we developed a theory which allows us to find the transition probabilities between the states of the Zeeman multiplet in the presence of the regular timedependent and random magnetic field (noise). The solution of this problem may be possible since the evolution matrix for the quantum problem is a rotation matrix acting in a spin $S$ representation. The density matrix can be expanded into a linear superposition of irreducible tensor operators. The coefficients at this operators related to one of the irreducible representations (Bloch tensors) evolve independently on others. Thus, the initial problem in the space of dimensionality $(2 S+1) \times(2 S+1)$ is reduced to $2 S$ separate problems in the linear spaces of dimensionality from 1 to $2 S$.

The second key observation is that, for the fast noise, the transitions due to the noise and those due to the regular part of magnetic field are separated in time. This fact allows us to solve the problems for regular field and for the noise in the absence of the regular nondiagonal field separately and then match them. An interesting conclusion of our theory is that, in contrast to usual statistical calculations with the white noise, in which only the Fourier component of the noise correlation function with zero frequency matters, the transition probabilities in the Landau-Zener problem depend only on the average square of the random field amplitude. We were able to find asymptotically exact analytical results for the probabilities. From them we concluded that, in the absence of initial coherence, the average values of the diagonal components of the Bloch tensors (with zero projection onto the direction of sweeping field) decrease monotonously with time due to the noise. It means that the population differences in average can only decrease after the transitions. However, they can grow if there is a coherence in the initial state and nondiagonal components of the Bloch tensors are not zero. Due to high symmetry, the considered system has $2 S$ additional integrals of motion: traces of the square of each Bloch tensor. Thus, the increase of the population differences proceeds at the expense of the non-diagonal components, i.e. coherence amplitudes and vice versa.

The same conservation laws enabled us to find exactly the fluctuations of the Bloch's tensors, in particular the fluctuations of the transition probabilities in the genuine LandauZener problem. They are of the same order of magnitude that the average values.

The noise in our theory is considered as the classical random field. To incorporate the quantum properties of noise is an interesting and challenging problem. The second unsolved problem is to study the correlation of the Bloch tensors at different moments of time.

\section{ACKNOWLEDGMENT}

This work was supported by NSF under Grant Nos. DMR 0072115, DMR 0103455, and DMR 0321572, by DOE under Grant No. DE-FG03-96ER45598, and by the Telecommunication and Informatics Task Force at Texas A\&M University. V.P. acknowledges the support from the Humboldt Foundation and Professor Thomas Nattermann and University of Cologne, Germany for the hospitality extended to him at final stage of this work.

\section{APPENDIX}

Here we present several lowest operator spherical harmonics $T_{s, m}^{S}$. In contrast to scalar spherical harmonics, they depend on three parameters: two of them are integers ( $s$ and $m$ ), whereas $S$ accepts integer and half-integer values. The simplest nontrivial harmonics are vectors with $s=1$. They are

$$
T_{1,0}^{S}=S_{z} ; T_{1, \pm 1}^{S}=\frac{1}{\sqrt{2}} S_{ \pm} .
$$

Next we demonstrate second-order tensorial harmonics:

$$
\begin{gathered}
T_{2, \pm 2}^{S}=\frac{1}{2} S_{ \pm}^{2} ; T_{2, \pm 1}^{S}=\frac{1}{2}\left(S_{ \pm} S_{z}+S_{z} S_{ \pm}\right) ; \\
T_{2,0}^{S}=\sqrt{\frac{3}{2}}\left[S_{z}^{2}-\frac{1}{3} S(S+1)\right] .
\end{gathered}
$$

The third rank harmonics read

$$
\begin{gathered}
T_{3, \pm 3}^{S}=\frac{1}{2^{3 / 2}} S_{ \pm}^{3} ; \quad T_{3, \pm 2}^{S}=\frac{1}{\sqrt{6}}\left(S_{ \pm}^{2} S_{z}+S_{ \pm} S_{z} S_{ \pm}+S_{z} S_{ \pm}^{2}\right), \\
T_{3, \pm 1}^{S}=\sqrt{\frac{5}{12}}\left[S_{z}^{2} S_{+}+S_{z} S_{+} S_{z}+S_{+} S_{z}^{2}-\frac{3 S(S+1)-1}{5} S_{+}\right], \\
T_{3,0}^{S}=\sqrt{\frac{5}{2}}\left[S_{z}^{3}-\frac{3 S(S+1)-1}{5} S_{z}\right] .
\end{gathered}
$$

For the fourth-rank harmonics we find

$$
\begin{gathered}
T_{4, \pm 4}^{S}=\frac{1}{4} S_{ \pm}^{4}, \\
T_{4, \pm 3}^{S}=\frac{1}{2^{5 / 2}}\left(S_{ \pm}^{3} S_{z}+S_{ \pm}^{2} S_{z} S_{ \pm}+S_{ \pm} S_{z} S_{ \pm}^{2}+S_{z} S_{ \pm}^{3}\right), \\
T_{4, \pm 2}^{S}=\frac{\sqrt{7}}{12}\left[S_{z}^{2} S_{ \pm}^{2}+S_{z} S_{ \pm} S_{z} S_{ \pm}+S_{z} S_{ \pm}^{2} S_{z}+S_{ \pm} S_{z}^{2} S_{ \pm}\right. \\
\left.+S_{ \pm} S_{z} S_{ \pm} S_{z}+S_{ \pm}^{2} S_{z}^{2}-\kappa S_{ \pm}^{2}\right],
\end{gathered}
$$




$$
\begin{gathered}
T_{4, \pm 1}^{S}=\frac{\sqrt{7}}{2^{5 / 2}}\left[S_{z}^{3} S_{ \pm}+S_{z}^{2} S_{ \pm} S_{z}+S_{z} S_{ \pm} S_{z}^{2}+S_{ \pm} S_{z}^{3}\right. \\
\left.-\kappa\left(S_{z} S_{ \pm}+S_{ \pm} S_{z}\right)\right], \\
T_{4,0}^{S}=\frac{\sqrt{35}}{4}\left(S_{z}^{4}-\kappa S_{z}^{2}+\lambda\right),
\end{gathered}
$$

where we have introduced the notation

$$
\kappa=\frac{6 S(S+1)-5}{7}, \quad \lambda=\frac{3 S(S+1)[S(S+1)-2]}{35} .
$$

${ }^{1}$ W. Wernsdorfer and R. Sessoli, Science 284, 133 (1999).

${ }^{2}$ VV. Dobrovitski and AK. Zvezdin, Europhys. Lett. 38, 377 (1997).

${ }^{3}$ K. Saito, S. Miyashita, and H. De Raedt, Phys. Rev. B 60, 14553 (1999).

${ }^{4}$ N.V. Prokof'ev and P.C.E. Stamp, Phys. Rev. Lett. 80, 5794 (1998).

${ }^{5}$ L.D. Landau, Phys. Z. Sowjetunion 2, 46 (1932).

${ }^{6}$ C. Zener, Proc. R. Soc. London, Ser. A 137, 696 (1932).

${ }^{7}$ W. Wernsdorfer et al., Europhys. Lett. 50, 552 (2000).

${ }^{8}$ E. Kececioglu and A. Garg, Phys. Rev. B 63, 064422 (2001).

${ }^{9}$ N. Sinitsyn and N. Prokof'ev, Phys. Rev. B 67, 134403 (2003).

${ }^{10}$ E.M. Chudnovsky and X. Martinez-Hidalgo, Phys. Rev. B 66, 054412 (2002).

${ }^{11}$ J. Larionova, M. Gross, M. Pilkington, H. Andres, H. StoeckiEvans, H.U. Gade, and S. Decurtnis, Angew. Chem., Int. Ed. Engl. 39, 1605 (2002).

${ }^{12}$ K. Dunbar and E. Shelter (private communication).

${ }^{13}$ F.T. Hioe, J. Opt. Soc. Am. B 4, 1327 (1987); C.E. Carroll, F.T. Hioe, J. Phys. A 19, 1151 (1986).
${ }^{14}$ V.L. Pokrovsky and N.A. Sinitsyn, cond-mat/0012303 (unpublished).

${ }^{15}$ Y. Kayanuma, J. Phys. Soc. Jpn. 53, 108 (1984); 54, 2037 (1985).

${ }^{16}$ V.L. Pokrovsky and N.A. Sinitsyn, Phys. Rev. B 67, 144303 (2003).

${ }^{17}$ E.M. Lifshitz and L.D. Landau, "Quantum Mechanics: NonRelativistic Theory" V3 (Butterworth-Heinemann, 1981).

${ }^{18}$ N. Vilenkin, A. Klimyk, Representation of Lie group and Special Function (Kluwer, Dordrecht, 1991), Vols. 1-3.

${ }^{19}$ A. Erdlyi, W. Magnus, F. Oberhettinger, and F. G. Tricomi, Higher Transcendental Functions (Krieger, Malabar, FL, 1981).

${ }^{20}$ A.A. Abrikosov, L.P. Gorkov, and I.E. Dzyaloshinskii, Methods of Quantum Field Theory in Statistical Physics (Prentice Hall, New York, 1963).

${ }^{21}$ N.F. Bell, R.F. Sawyer, R.R. Volkas, and Yv.Y.Y. Wong, Phys. Rev. A 65, 042328 (2002).

${ }^{22}$ I. Chiorescu, W. Wernsdorfer, A. Mller, S. Miyashita, and B. Barbara, Phys. Rev. B 67, 020402(R) (2003).

${ }^{23}$ M. Nishino, K. Saito, and S. Miyashita, Phys. Rev. B 65, 014403 (2002). 